\title{
COMPORTAMENTO SILVICULTURAL E DANOS EM MOGNO- AFRICANO (Khaya ivorensis A. Chev.) EM SISTEMA SILVIPASTORIL
}

\author{
SILVICULTURAL BEHAVIOR AND DAMAGE IN AFRICAN MAHOGANY \\ (Khaya ivorensis A. Chev.) IN SILVIPASTORAL SYSTEM \\ Rubens Marques Rondon Neto ${ }^{1}$, Rhuan Pierri Pachinski Kruger ${ }^{1}$, \\ Ana Karolina Queiroz Rodrigues ${ }^{1}$ \\ ${ }^{1}$ Universidade do Estado de Mato Grosso, Alta Floresta, Mato Grosso, Brasil - \\ rubensrondon@yahoo.com.br, anakarolinarodrigues1502@gmail.com \& rhuankruger@hotmail.com
}

\begin{abstract}
RESUMO
O objetivo deste trabalho foi avaliar o comportamento silvicultural e os danos nas cascas das árvores de mognoafricano (Khaya ivorensis A. Chev.) causados por bovinos em sistema silvipastoril, em Paranaíta/MT. Os modelos de plantios avaliados foram: 1) Modelo 1: linhas simples de $K$. ivorensis, no espaçamento $6 \times 35 \mathrm{~m}$; e 2) Modelo 2: linhas simples de eucalipto urograndis (Eucalyptus uroplhylla $\times$ E. grandis) associado com $K$. ivorensis, na proporção 3:1, no espaçamento $4 \times 35 \mathrm{~m}$. Em três parcelas foram amostrados um total de 60 e 36 indivíduos no modelo de plantio 1 e 2, respectivamente, mas foram selecionados 30 indivíduos aleatoriamente para análises estatísticas. Foram avaliadas as taxas de sobrevivência, crescimento em altura e diâmetro e área de copa. Também foram avaliados os danos nas cascas do tronco das árvores causadas pelos bovinos. Tais dados foram analisados estatisticamente pelo teste não paramétrico de Kruskal-Wallis, posteriormente foi aplicado o teste de Dunn. As médias das taxas de sobrevivência de $K$. ivorensis no modelo de plantio 1 foi de $86,5 \%$, enquanto no modelo de plantio 2 cerca de $95,0 \%$ dos indivíduos se encontravam vivos. Em ambos os modelos de plantios todos os indivíduos de $K$. ivorensis sofreram algum tipo de lesão na casca do tronco causada pelos bovinos. Os danos na casca do tronco das árvores de $K$. ivorensis foram intensos independente do modelo de plantio em sistema silvipastoril, o que comprometeu o crescimento das árvores.
\end{abstract}

PALAVRAS-CHAVE: Arborização de pastagem, Integração pecuária-floresta, Sistema agroflorestal.

\section{ABSTRACT}

The objective of this work was to evaluate the silvicultural behavior and the damage to the bark of African mahogany (Khaya ivorensis A. Chev.) Trees caused by cattle in the silvopastoral system, in Paranaíta - Mato Grosso state (Brazil). The planting models evaluated were: 1) Model 1: simple lines of $K$. ivorensis, in the $6 \times 35 \mathrm{~m}$ spacing; and 2) Model 2: simple lines of eucalyptus urograndis (Eucalyptus uroplhylla $\times$ E. grandis) associated with $K$. ivorensis, in a 3: 1 ratio, in the $4 \times 35$ m spacing. In three plots, a total of 60 and 36 individuals were sampled in planting model 1 and 2, respectively, but 30 individuals were randomly selected for statistical analysis. Survival rates, growth in height and diameter and canopy area were evaluated. The damage to the tree bark caused by cattle was also evaluated. Such data were analyzed statistically by the Kruskal-Wallis non-parametric test, after which the Dunn test was applied. The average survival rates for $K$. ivorensis in the planting model 1 was $86.5 \%$, while in the planting model 2 about $95.0 \%$ of the individuals were alive. In both planting models, all individuals of $K$. ivorensis suffered some type of lesion on the trunk bark caused by cattle. The damage to the bark of the tree of $K$. ivorensis was intense regardless of the planting model in silvopastoral system, which compromised the growth of the trees.

KEYWORDS: Pasture afforestation, Livestock-forest integration, Agroforestry system. 


\section{INTRODUÇÃO}

No ano de 2019 o rebanho bovino brasileiro era de aproximadamente 213,676 milhões de cabeças, sendo que o Mato Grosso contribuiu com cerca de 29,873 milhões de cabeças de gado, ou seja, 13,98\% do total do rebanho do país (ABIEC, 2020). Enquanto que a área total ocupada com pastagem em terras mato-grossense era de 21.260.587 ha, o que equivale a $14,2 \%$ do total existente no Brasil (149.670.217 ha) (BRASIL, 2018). Frente a esses dados percebe-se a importância da atividade de bovinocultura no país e no Estado de Mato Grosso. No entanto, ainda é possível de diversificar a produção e incrementar a produtividade animal, através da implantação e manejo de diferentes modelos de sistemas silvipastoris.

Os sistemas silvipastoris são constituídos pelo arranjo espacial e temporal do componente arbóreo, animal e a planta forrageira dentro do sistema produtivo agroflorestal. A combinação dos componentes é feita de forma planejada, a fim de incrementar os benefícios ecológicos e econômicos das interações entre eles. Dentre os diversos benefícios dos sistemas silvipastoris merece destaque no Estado de Mato Grosso a melhoria do microclima das áreas de pastagens. As sombras das árvores nas pastagens promovem o conforto térmico para o gado durante o ano inteiro, principalmente nas horas mais quentes do dia. Além disso, há uma demanda presente e crescente de madeira para uso nas benfeitorias da propriedade rural, principalmente nas construções e reformas de cercas, porteiras e currais, além da oportunidade de comercialização da madeira serrada nas indústrias madeireiras e moveleiras.

Acredita-se que um dos desafios dos variados modelos de sistemas silvipastoris diz respeito ao domínio da complexidade das inter-relações entre as espécies arbóreas $x$ forrageiras $x$ animais. $O$ fato é devido à dificuldade técnica de definição do arranjo espacial e temporal de cada componente no modelo de sistema silvipastoril, obtendo o máximo de resultados positivos de cada elemento produtivo. Os danos dos animais nas cascas do tronco das árvores é um exemplo de desarmonia entre os elementos do sistema de produção agroflorestal. Tal situação pode inviabilizar os resultados esperados do modelo de sistema silvipastoril adotado, afetando a qualidade da madeira, o crescimento e o estado fitossanitário ou até mesmo provocar a morte das árvores.

Aliada a questão estrutural dos modelos de sistemas silvipastoris tem-se ainda a seleção do componente arbóreo como sendo fundamental, pois a espécie florestal escolhida deve se adaptar às condições que o sistema de produção integrado proporciona. Dentre as diversas espécies arbóreas que apresentam madeira de alto valor econômico no mercado interno e externo, com potencial de usos em sistemas silvipastoris, surge como alternativa o mogno-africano (Khaya ivorensis A. Chev.). Segundo Ribeiro et al. (2019) trata-se de uma espécie florestal nativa da África Ocidental, que produz madeira nobre de alto valor comercial. A área estimada de plantio no Brasil no ano de 2018 era de aproximadamente 37 mil ha, em povoamentos puros e em sistemas integrados.

Nesse contexto, o presente trabalho teve por objetivo avaliar o comportamento silvicultural e os danos nas cascas das árvores de mogno-africano (Khaya ivorensis A. Chev.) causados por bovinos em sistema silvipastoril, instalado no município de Paranaíta/MT.

\section{MATERIAL E MÉTODOS}

A pesquisa foi desenvolvida em um sistema agroflorestal do tipo silvipastoril, pertencente à Estância Vanda, situada no município de Paranaíta - extremo norte do Estado de Mato Grosso. A área total de do sistema de produção é de aproximadamente 24 ha. O local de estudo encontra-se situado entre as coordenadas geográficas $9^{\circ} 40^{\prime} 23^{\prime \prime} \mathrm{S}$ e $56^{\circ} 28^{\prime} 50^{\prime \prime} \mathrm{W}$ e a aproximadamente $267 \mathrm{~m}$ de altitude em relação ao nível do mar.

Pela classificação de Köppen, o clima da região da área experimental é do tipo Am com temperatura média de 26으 e precipitação anual cerca de $2200 \mathrm{~mm}$ (ALVARES et al., 2013). O solo predominante na região é classificado como Latossolo Vermelho-Amarelo e relevo suave ondulado (MOREIRA \&VASCONCELOS, 2007). A vegetação original se caracteriza como Floresta Ombrófila Aberta (IBGE, 2012)

Os dois modelos de sistema silvipastoril é formado por linhas simples e puras de mogno-africano (Khaya ivorensis) e também linhas combinadas com eucalipto urograndis (híbrido de Eucalyptus urophylla x E. grandis) (Figura 1). Nos dois primeiros anos de plantio as linhas das árvores foram isoladas com cerca elétrica. Cerca de 60 cabeças gado das misturas entre as raças jersey $e$ holandesa (jersolando) seguiam o pastoreio rotacionado nas áreas dos sistemas silvipastoris. Em ambos os modelos de plantio florestal houve a mesma intensidade de animais por área ( 6 - 10 cabeças $\left.h^{-1}\right)$ e tempo de pastejo (25 a 35 dias). Abaixo seguem os detalhes dos arranjos espaciais e temporais dos componentes arbóreos associado com pastagem de Panicum maximum cv. 
mombaça nos dois sistemas silvipastoris avaliados, sendo:

- Modelo de plantio 1: linhas simples de $K$. ivorensis, no espaçamento $35 \times 6 \mathrm{~m}$ (48 indivíduos ha-1). Implantado em 2014;

- Modelo de plantio 2: linhas simples de K. ivorensis e E. urograndis associado com, na proporção 1:3, no espaçamento $35 \times 4 \mathrm{~m},(24$ e 47 indivíduos ha-1, respectivamente). Implantado em 2015.
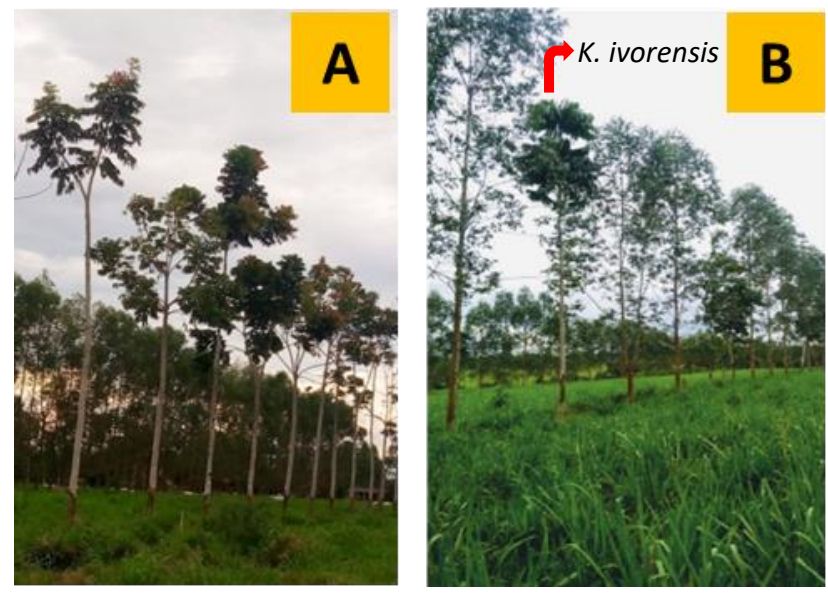

Figura 1. Modelos de plantio de K. ivorensis em sistema silvipastoril com linhas simples e puras (A) e linhas simples de $K$. ivorensis associadas com Eucalyptus urophylla $\times$ E. grandis (B).

No arranjo espacial de $K$. ivorensis em linhas simples foram amostradas três parcelas, cada uma com 20 árvores, totalizando-se 60 individuos arbóreos. No segundo modelo de plantio, devido à baixa frequência de indivíduos de $K$. ivorensis foram medidas três parcelas de 12 árvores, que totalizou 48 árvores. Para a realização das análises estatísticas das variáveis dendrométricas e as relacionadas aos danos nas cascas das árvores foram selecionados 30 indivíduos de forma aleatória em cada modelo de plantio.

A coleta dos dados ocorreu durante os meses de fevereiro e março de 2019. As variáveis dendrométricas avaliadas foram: circunferência a 1,3 $\mathrm{m}$ de altura do solo (CAP), crescimento em altura total, diâmetro de copa (sentido N-S e E-W), altura da primeira bifurcação do fuste (altura comercial). O CAP foi mensurado utilizando uma fita métrica, as duas alturas com o auxílio do hipsômetro Blume Leiss e os diâmetros de copa foram medidos com trena. Também foi estimada a taxa de sobrevivência dos indivíduos em cada modelo de plantio.

Também foi avaliada a proporção de danos nas cascas das árvores causados por bovinos através do método gravimétrico. Inicialmente, as lesões do tronco foram contornadas em folhas de papel jornal com o uso de giz de cera. Em seguida o papel era recortado e pesado em balança de precisão. Os pesos foram relacionados com o peso de uma área previamente estabelecida do mesmo papel utilizado em campo (Figura 2).

As alturas dos danos foram mensuradas por meio de uma trena, medindo desde o solo, até o início e termino da lesão. A cerca de $10 \mathrm{~cm}$ da parte inferior e superior da lesão foram medidos as espessuras de cascas, a fim de avaliar possíveis efeitos dos danos nas profundidades dos tecidos do ritidoma. Para tanto, foi utilizado o medidor de espessura de casca - modelo Barktax. A taxa de sobrevivência das árvores também foi avaliada nesse trabalho.
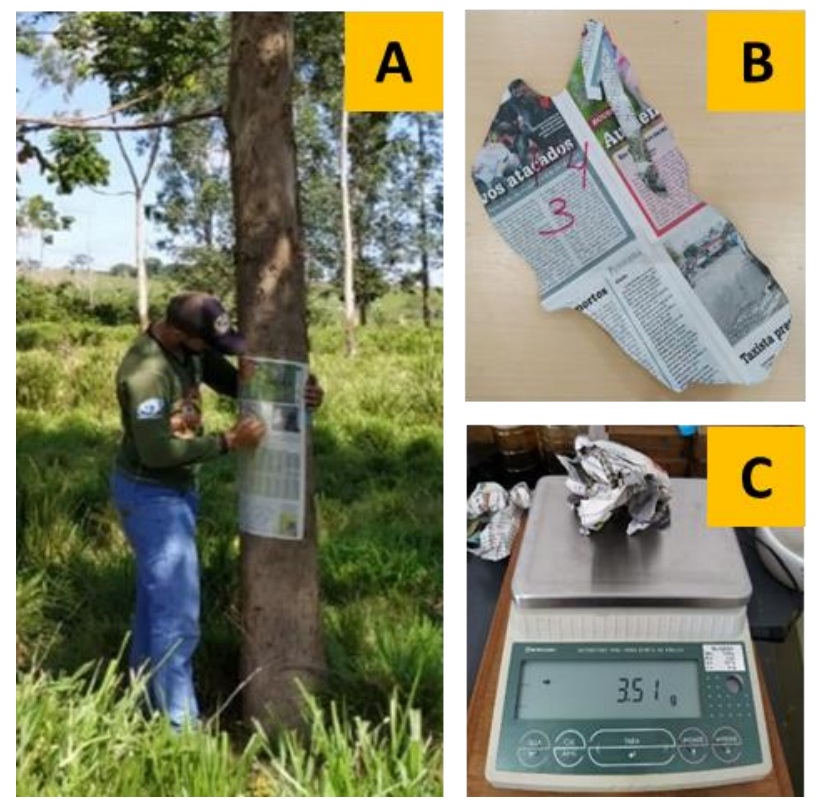

Figura 2. Medição da área de danos na casca do tronco das árvores de $K$. ivorensis causado por bovinos, iniciando com o contorno na área lesionada em papel $(\mathbf{A})$, recorte da área da lesão (B) e pesagem do papel recortado (C).

A área de projeção da copa no solo foi calculada por meio da (Equação 01), Azevedo \& Francelino (2011), sendo:

$$
C i=\pi *\left(\frac{L 1+L 2}{4}\right)^{2}
$$

Em que: $\mathrm{Ci}=$ Área da projeção individual da copa, fórmula de área da elipse $\left(\mathrm{m}^{2}\right) ; L 1=$ Comprimento da maior linha longitudinal da copa $(\mathrm{m})$; e $L 2=$ Comprimento da maior linha perpendicular à da copa (m).

Também serão realizadas as estimativas do incremento médio anual de altura total e comercial, DAP e área de copa, através da (Equação 02) dada por Imaña \& Encinas (2008). 


$$
I M A=\frac{Y f-Y i}{T}
$$

Em que: $I M A=$ incremento médio anual; $Y f=$ variável ao final da avaliação; $Y i$ = variável no início da avaliação; e $T=$ idade do indivíduo.

Os resultados primeiramente foram submetidos à análise de pressuposições básicas de normalidade e homocedasticidade, utilizando os testes de Shapiro-Wilk e Bartlett. Para os casos não atendidos, mesmo após transformação, foi utilizado o teste não paramétrico de Kruskal-Wallis, posteriormente foi aplicado o teste de Dunn. Deve se ressaltar a diferença de idade entre os modelos, logo os valores de incremento médio anual tornam-se interessantes para a avaliação.

\section{RESULTADOS E DISCUSSÃO}

As médias das taxas de sobrevivência de $K$. ivorensis no modelo de plantio 1 e 2 estudados foram iguais a $86,5 \%$ e $95,0 \%$, respectivamente, não havendo diferenças estatísticas entre elas. Em um sistema de integração lavoura-pecuária floresta (ILPF) implantado no município de Terra Alta/PA, após três anos de plantio, Sales et al. (2017) obtiveram valor médio de sobrevivência de $K$. ivorensis semelhantes aos encontrados neste trabalho $(93,8 \%)$.

O motivo da maior taxa de sobrevivência no modelo de plantio 2 pode ser sugerida devido ao fato de haver maior dificuldade dos animais em encontrar os indivíduos de $K$. ivorensis quando associados ao $E$. urograndis. Dessa forma, a mistura de outras espécies florestais nas linhas de plantio com $K$. ivorensis pode ter sido uma estratégia de minimizar os índices de danos no tronco das árvores. No entanto, em condições de campo observou-se que ao longo do tempo há possibilidade de aumento dos índices de mortalidade das árvores de $K$. ivorensis em decorrência do agravamento das lesões nas cascas do tronco ocasionadas pela predação de bovinos.

As médias de altura total $(\mathrm{Ht})$ e comercial $(\mathrm{Hc})$ foram maiores no modelo de plantio 1 , apresentando diferenças estatísticas dos resultados obtidos no sistema de plantio 2 (Tabela 1). Os respectivos valores médios de $\mathrm{Ht}$ e $\mathrm{Hc}$ no modelo de plantio 1 foram superiores em cerca de $42,6 \%$ e $37,0 \%$ em relação ao modelo 2 . O fato pode ser atribuído às situações desiguais de competições por espaços entre os indivíduos nos modelos de plantios analisados, além da diferença de idade de implantação de cada sistema silvipastoril.

Os respectivos incrementos em altura total (IMA de $\mathrm{Ht}$ ) e comercial (IMA de $\mathrm{Hc}$ ) no modelo de plantio 1 foram maiores em aproximadamente de $21,1 \%$ e $28,7 \%$ em comparação ao modelo de plantio 2. Resultados de IMA de $\mathrm{Ht}$ superiores aos encontrados neste estudo foram obtidos por Ribeiro et al. (2017), em plantios puros de $K$. ivorensis, localizados em Pirapora/MG. Para Schneider (2004) o crescimento em altura é afetado predominantemente pela qualidade do sítio e não pela concorrência entre os indivíduos.

Tabela 1. Valores das médias e desvios padrão da altura total $(\mathrm{Ht})$ e comercial $(\mathrm{Hc})$, DAP e área de copa $(\mathrm{Ac})$, com os respectivos incrementos médios anuais (IMA) de $K$. ivorensis em diferentes modelos de plantio no sistema silvipastoril.

\begin{tabular}{ccc}
\hline Variáveis & \multicolumn{2}{c}{ Modelo de plantio } \\
\cline { 2 - 3 } dendrométricas & $\mathbf{1}$ & $\mathbf{2}$ \\
\hline $\mathrm{Ht}(\mathrm{m})$ & $13,02 \pm 1,96 \mathrm{a}$ & $8,20 \pm 1,86 \mathrm{~b}$ \\
$\mathrm{Hc}(\mathrm{m})$ & $8,68 \pm 2,51 \mathrm{a}$ & $4,98 \pm 1,68 \mathrm{~b}$ \\
$\mathrm{DAP}(\mathrm{cm})$ & $18,31 \pm 2,80 \mathrm{a}$ & $15,26 \pm 2,48 \mathrm{~b}$ \\
$\mathrm{Ac}\left(\mathrm{m}^{2}\right)$ & $13,11 \pm 10,30 \mathrm{a}$ & $4,70 \pm 3,85 \mathrm{~b}$ \\
IMA de $\mathrm{Ht}(\mathrm{m})$ & $2,60 \pm 0,39 \mathrm{a}$ & $2,05 \pm 0,46 \mathrm{~b}$ \\
\hline IMA de $\mathrm{Hc}(\mathrm{m})$ & $1,74 \pm 0,50 \mathrm{a}$ & $1,24 \pm 0,42 \mathrm{~b}$ \\
IMA de DAP $\left(\mathrm{cm}^{\mathrm{ano}} \mathrm{an}^{-1}\right)$ & $3,66 \pm 0,56 \mathrm{a}$ & $3,81 \pm 0,62 \mathrm{a}$ \\
\hline IMA de Ac $\left(\mathrm{m}^{2}\right.$ ano $\left.^{-1}\right)$ & $4,70 \pm 3,85 \mathrm{~b}$ & $1,17 \pm 0,96 \mathrm{~b}$ \\
\hline
\end{tabular}

Médias seguidas de mesma letra na coluna não diferem pelo pós-teste de Dunn ao nível de $5 \%$.

Quanto ao crescimento diamétrico (DAP) de $K$. ivorensis foi observado que houve diferenças estatísticas entre os modelos de plantio. A média de DAP encontrada no modelo de plantio 1 foi cerca de $16,6 \%$ maior do que o modelo de plantio 2. As médias de IMA de DAP dos dois modelos de plantio não diferiram estatisticamente entre si. Valor de IMA de DAP semelhante foram obtidos por Castro et al. (2008), em árvores de $K$. ivorensis implantadas em sistema silvipastoril em Benevides/PA, aos sete anos de idade.

$A$ área de copa $(A c)$ teve valores médios que diferiram estatisticamente entre os dois modelos de plantios avaliados. As respectivas médias de Ac e IMA de Ac no sistema de plantio 1 foi em torno de $35,8 \%$ e 55,3\% maior que a encontrada no modelo de plantio 2 . Outro ponto a ser considerado refere-se aos diâmetros médios de copa das árvores no sistema de plantio 1 e 2, que foram de 4,05 e 2,29 m, respectivamente. Sento assim, é possível perceber que as árvores de $E$. urograndis foram mais eficientes em ocupar os espaços com suas copas nas linhas de plantio do modelo 2.

A superioridade dos valores médios dos parâmetros dendrométricos de $K$. ivorensis no modelo de plantio 1 pode ser devido ao seu maior tempo de implantação. Outro aspecto importante a ser considerado diz respeito à 
competição mais equilibrada por recursos nas linhas de plantio puras, principalmente pela luz. Assim sendo, os indivíduos crescem de forma semelhante, não havendo muitas diferenças de crescimento ao ponto de uma árvore suprimir a outra. Portanto, acredita-se que os modelos de plantio com linhas simples e puras podem ser interessantes para evitar a supressão de árvores, desuniformidade de crescimento em altura e diâmetro e até mesmo aumento das taxas de mortalidade ao longo do tempo.

Os resultados referentes à espessura de casca do tronco, área lesionada e altura dos danos podem ser observados na Tabela 2. As espessuras das cascas nas partes inferiores e superiores às lesões não apresentaram diferenças estatísticas nos dois modelos de plantio testados. Nos sistemas de plantios 1 e 2 as lesões feitas pelos bovinos se estenderam de $0,37-1,75 \mathrm{~m}$ e $0,43-$ $1,87 \mathrm{~m}$, respectivamente., mas não diferiram estatisticamente. Essas alturas das lesões são úteis na determinação das alturas dos objetos de proteção individual das árvores em plantios feitos com a presença do gado.

Tabela 2. Média e desvio padrão da espessura de casca, altura e área dos danos na casca das árvores de $K$. ivorensis em diferentes modelos de plantio no sistema silvipastoril.

\begin{tabular}{ccc}
\hline Variáveis dendrométricas & \multicolumn{2}{c}{ Modelo de plantio } \\
\cline { 2 - 3 } & $\mathbf{1}$ & $\mathbf{2}$ \\
\hline $\begin{array}{c}\text { Espessura da casca da } \\
\text { parte inferior }(\mathrm{cm})\end{array}$ & $1,07 \pm 0,12 \mathrm{a}$ & $0,94 \pm 0,11 \mathrm{a}$ \\
$\begin{array}{c}\text { Espessura da casca da } \\
\text { parte superior }(\mathrm{cm})\end{array}$ & $0,86 \pm 0,11 \mathrm{a}$ & $0,84 \pm 0,09 \mathrm{a}$ \\
$\begin{array}{c}\text { Altura do dano da } \\
\text { parte inferior }(\mathrm{m})\end{array}$ & $0,55 \pm 0,18 \mathrm{a}$ & $0,67 \pm 0,24 \mathrm{~b}$ \\
$\begin{array}{c}\text { Altura do dano da } \\
\text { parte superior }(\mathrm{m})\end{array}$ & $1,66 \pm 0,09 \mathrm{a}$ & $1,72 \pm 0,15 \mathrm{~b}$ \\
Área de dano na casca $\left(\mathrm{m}^{2}\right)$ & $0,30 \pm 0,11 \mathrm{a}$ & $0,13 \pm 0,06 \mathrm{~b}$ \\
\hline
\end{tabular}

Médias seguidas de mesma letra na coluna não diferem pelo pós-teste de Dunn ao nível de $5 \%$.

Em ambos os modelos de plantio de $K$. ivorensis avaliados todos os indivíduos amostrados sofreram danos na casca do tronco ocasionada pela predação de bovinos (Figura 3). Todas as lesões alcançaram o lenho das árvores, retirando parte do tecido cambial, no entanto, não houveram danos nas copas devido à eficiência no isolamento feito pela cerca elétrica. Conforme Silva et al. (2019) os bovinos também causaram danos no tronco de todas as árvores de Khaya grandifoliola, em um sistema silvipastoril implantado em Araputanga/MT. Altos índices de danos na casca do tronco de $K$. ivorensis que foi danificada pelo gado também foram obtidos por Silva et al. (2017), em um ILPF implantado em Paragominas/PA.

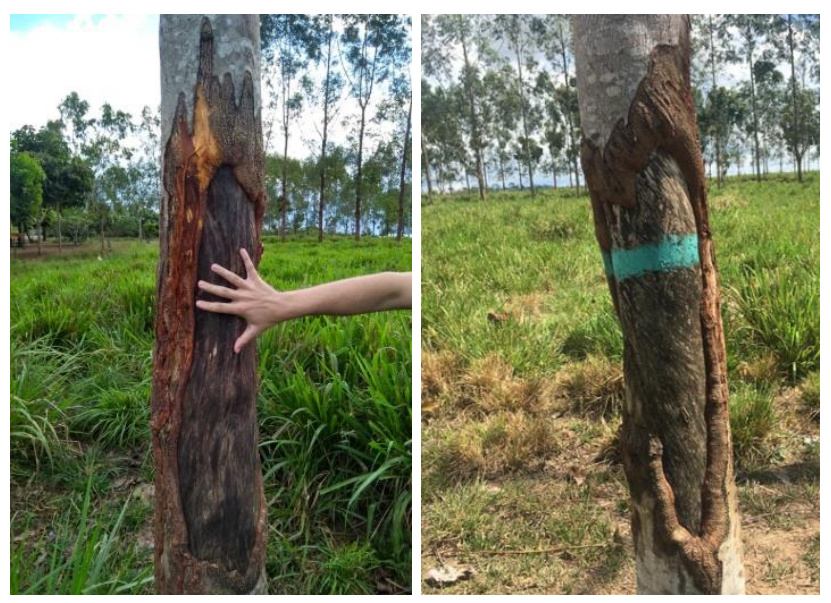

Figura 3. Danos na casca do tronco de $K$. ivorensis causados por bovinos em sistema silvipastoril.

As áreas dos danos nas cascas dos troncos das árvores de $K$. ivorensis variaram de 0,19-0,41 $\mathrm{m}^{2}$ e 0,07-0,19 $\mathrm{m}^{2}$ no modelo de plantio 1 e 2 , respectivamente. $A$ área média da lesão no modelo de plantio 1 foi cerca de $56,7 \%$ superior aos prejuízos obtidos no modelo de plantio 2, diferenciando estatisticamente. A fim de cicatrizar as lesões sofridas pelos bovinos percebeu-se em campo a formação de calos de cicatrização nas regiões laterais. Em um sistema de ILPF com linhas simples de $K$. ivorensis, Falesi \& Baena (1999) verificaram que $18,1 \%$ dos indivíduos tinham danos maiores que $5 \mathrm{~cm}$ de diâmetro, $39,2 \%$ apresentavam lesão de casca, sem alcançar o câmbio, e $26,2 \%$ das árvores mostravam lesões no tronco alcançando o lenho pela retirada do tecido cambial.

A ocorrência de danos nas cascas das árvores de $K$. ivorensis pode contribuir com o aumento das taxas de mortalidade ao longo do tempo, pois há perda da parte essencial de suas defesas contra agentes xilófagos e patogênicos. Outro aspecto importante a ser considerado refere-se aos tamanhos das lesões, as quais dificilmente serão cicatrizadas, podendo provocar apodrecimento do lenho e consequentemente a morte dos indivíduos mais danificados.

Vale ressaltar que a escolha de $K$. ivorensis para compor os sistemas silvipastoris teve como objetivo produzir madeira serrada e disponibilizar sombra para o gado. Portanto, os danos no tronco das árvores não foram desejáveis, pois o componente florestal perderá seu valor comercial, devido apresentar defeitos ao longo do tronco. Assim sendo, o grau dos danos no troco dos indivíduos de $K$. ivorensis foi determinante para o sucesso ecológico e econômico da interação dos componentes que compõem os dois sistemas silvipastoris, especialmente quando a 
produção madeireira é considerada muito importante.

A fim de encontrar os possíveis motivos para a predação das cascas das árvores de $K$. ivorensis causados pelos bovinos, observou-se em campo o controle satisfatório de ecto e endoparasitas, ausência de estresse social entre os animais e carga animal adequada nas pastagens. No entanto, apesar da boa disponibilidade de forragem e sal mineral não foi avaliada suas qualidades, a fim de diagnosticar possíveis desequilíbrios nutricionais nos animais. Entretanto, sugere-se que avaliação do comportamento dos animais criados nas pastagens formadas em sistemas silvipastoris é um pré-requisito para o sucesso do sistema de produção animal e florestal de forma integrada.

\section{CONCLUSÕES}

1 - Os danos na casca do tronco das árvores de $K$. ivorensis causadas por bovinos foram intensos independente do modelo de plantio em sistema silvipastoril.

2 - As intensidades das lesões nas cascas comprometeram o comportamento silvicultural da espécie florestal nos modelos de plantios integrados avaliados.

\section{REFERÊNCIAS}

ABIEC. Perfil da pecuária no Brasil: relatório anual. São Paulo: Beef Report, 2020.

ALVARES, C.A. et al. Köppen's climate classification map for Brazil. Meteorologische Zeitschrift, v.22, n.6, p.711-728, 2013.

AZEVEDO, V.K.; FRANCELINO, M.R. Determinação da cobertura de copa utilizando diferentes métodos. 19 Congresso Brasileiro de Reflorestamento Ambiental, 2011.

BRASIL. Censo Agropecuário 2017: resultados preliminares. Rio de Janeiro: IBGE, 2018.

CASTRO, A.C. et al. Sistema silvipastoril na Amazônia: ferramenta para elevar o desempenho produtivo de búfalos. Ciência Rural, v.38, n.8, p.2395-2402, 2008.

FALESI. I.C.; BAENA, A.R.C. Mogno Africano (Khaya ivorensis A. Chev.) em sistema silvipastoril com leguminosa e revestimento natural do solo. Belém: Embrapa Amazônia Oriental, 1999.

IBGE. Manual técnico da vegetação brasileira: sistema fitogeográfico, inventário das formações florestais e campestres, técnicas e manejo de coleções botânicas, procedimentos para mapeamentos. Rio de janeiro: IBGEDiretoria de Geociências, 2012.

IMAÑA, J.; ENCINAS, O. Epidometría forestal. Unb: Faculdade de
Ciências Florestais e Ambientais, 2008.

MOREIRA, M.L.C; VASCONCELOS, T.N.N. Mato Grosso: solos e paisagens. Cuiabá: SEPLAN/MT, 2007.

REIS, C.A.F. et al. Mogno-africano (Khaya spp.): atualidades e perspectivas do cultivo no Brasil. Usos, importância econômica e perspectivas de mercado. Brasília: Embrapa, 2019.

RIBEIRO, A. et al. O cultivo do mogno africano (Khaya spp.) e o crescimento da atividade no Brasil. Floresta e ambiente, v.24, p.1-11, 2017.

SALES, A. et al. Desempenho do mogno-africano (Khaya ivorensis A. Chev.) no sistema ILPF em Terra Alta-PA. In: 4 Simpósio de Estudos e Pesquisas em Ciências Ambientais na Amazônia, 2017.

SCHNEIDER, P.R. Manejo florestal: planejamento da produção florestal. Santa Maria: UFSM, 2004.

SILVA, D.D.F. et al. Vulnerability of African mahogany to cattle predation in a silvipastural system. Pesquisa Agropecuária Brasileira. v.54, p.1-5, 2019.

SILVA, A.R. et al. Incidência de danos em espécies florestais causados por bovinos em sistema de Integração LavouraPecuária-Floresta. Belém: Embrapa Amazônia Oriental, 2017.

Recebido em 16-04-2021 Aceito em 11-05-2021 